\title{
ESTUDIO DE LAS COLOCACIONES EN EL ÁMBITO HISPÁNICO DURANTE LOS ÚLTIMOS VEINTE AÑOS ${ }^{1^{*}}$
}

\author{
Paula Martos García \\ Universidad de Granada \\ paulam@ugr.es
}

\section{RESUMEN}

En este artículo se analizan los trabajos centrados en el estudio de las colocaciones en el ámbito de la lengua española durante los últimos veinte años. La segmentación temporal obedece a que desde la última década del siglo xx asistimos a un aumento exponencial de las investigaciones sobre colocaciones, tanto desde la perspectiva sincrónica como diacrónica. Partiendo de la distinción entre los enfoques de corte estadístico y las aportaciones marcadas por un carácter semántico, repasamos las teorías más destacadas que se han ocupado de las colocaciones. Asimismo, nos aproximamos a una definición de colocación y repasamos algunos de sus rasgos característicos que permiten distinguirlas de otras combinaciones léxicas.

PALABRAS CLAVE: colocaciones; español; revisión; definición; rasgos

\section{ABSTRACT}

In this paper, we analyse the works focused on the study of collocations of the Spanish language during the last twenty years. This temporal segmentation is due to an exponential increase in studies on these structures, from both synchronous and diachronic perspectives in the last decade of $20^{\text {th }}$ century. Based on the distinction between statistical and semantic researches, we review the most important theories that have put the spotlight on collocations. Also, we approach a definition of collocation and review some of its characteristic features that distinguish them from other lexical combinations.

KEY WORDS: collocation; Spanish; review; definition; features

\footnotetext{
1* Este artículo se inscribe en el Proyecto de Excelencia P09HUM4466, (Junta de Andalucía / Fondos FEDER).
} 


\section{INTRODUCCIÓN ${ }^{2 * *}$}

Las colocaciones constituyen un tipo de combinación léxica marcado por una gran controversia. En palabras de Bosque (2004:XXII), se trata de "un concepto complejo, sumamente polémico, de límites difusos"; para Alonso Ramos (2004b:33-67), se hallan "a caballo entre el léxico y la sintaxis". Ante estas afirmaciones, se pone de manifiesto la necesidad de centrar la atención en esta clase de combinaciones léxicas con el fin de desentrañar, por un lado, el concepto de colocación y, por otro, especificar los rasgos atribuibles a las unidades que son consideradas como tales. Por ende, resulta imprescindible revisar las investigaciones que se han realizado sobre ellas en los últimos veinte años, teniendo en cuenta que es en este periodo en el que se incrementa la atención recibida por estas estructuras en los estudios sobre el español.

A pesar de que en la actualidad se considera de manera general que las colocaciones se incluyen entre las unidades fraseológicas (Corpas Pastor 1996, 2001, 2003; Castillo Carballo 2001:267; Moreno Pereiro y Buyse 2003; Gómez Molina 2004; Navarro 2003; Pazó y Travalia 2008; Ferrando Aramo 2012), existen opiniones en contra $^{3}$. La falta de consenso viene dada por la escasez de publicaciones especializadas y por la carencia de profundidad en su estudio, especialmente en el terreno de la lengua española.

\section{OBJETIVOS}

En el presente trabajo reflexionaremos sobre las diferentes teorías que se han ocupado de las colocaciones desde una perspectiva tanto sincrónica como diacrónica y analizaremos los criterios propuestos para reconocer estas estructuras lingüísticas. Asimismo, tras una revisión de las aportaciones más significativas en los últimos años, focalizaremos nuestra atención en la definición que, a nuestro juicio, resulta más acertada para el término colocación y en algunos de sus caracteres distintivos.

\section{ESTUDIOS SINCRÓNICOS}

Es el último decenio del siglo xx el momento en el que se dispara el interés por las colocaciones en el ámbito de la lengua española. Cabe recordar, no obstante, que las investigaciones sobre estas estructuras comenzaron varias décadas antes, a mediados del siglo xx. En el conjunto de las teorías acerca de ellas podemos disociar de manera nítida dos corrientes:

\footnotetext{
${ }^{2 * *}$ Para la realización de este trabajo han resultado imprescindibles las valiosas aportaciones del Doctor Calderón Campos y la Doctora Espinosa Elorza, a los que transmito mi más profundo agradecimiento.

${ }^{3}$ García-Page considera que tanto las estructuras que define como colocaciones como las construcciones con verbo de apoyo no son más que "expresiones libres", si bien no libérrimas, pues están también sujetas a ciertas restricciones semánticas"(2001:193). Para Wotjak (1998:268) aunque las colocaciones "gozan de cierto grado de predictibilidad", no pueden situarse en un estatus similar al de las unidades fraseológicas. Muñiz Álvarez (2002:266) se decanta también porque las colocaciones se hallan más cerca de las combinaciones libres que de las unidades fraseológicas.
} 
una basada en la frecuencia de coaparición de sus componentes (3.1) y otra fundamentada en los vínculos semánticos (3.2).

\subsection{Enfoque estadístico}

Como acabamos de mencionar, el estudio sobre las colocaciones tuvo su inicio a mediados de la centuria pasada, concretamente, en el seno del contextualismo británico, de la mano de Firth (1957:194), quien empleó por primera vez el término collocation en referencia a la coocurrencia frecuente de dos unidades léxicas (Alonso Ramos 1994-1995:9; Corpas Pastor

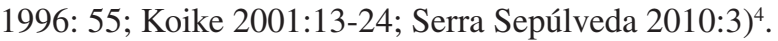

En el contextualismo se halla el origen de la primera teoría sobre las colocaciones, especialmente en lo que se refiere a la caracterización de estas unidades atendiendo a la frecuencia de coaparición de los elementos que las componen. La interpretación estadística de los datos permitió completar este enfoque, de gran aceptación entre los discípulos de Firth, Halliday $(1961,1966)$ y Sinclair $(1966,1991)$. El éxito de la perspectiva basada en la coocurrencia fue tal que, a partir de los trabajos en los que se toma como punto de partida el enfoque estadístico, se elaboró el Collins COBUILD English Language Dictionary (1987). No obstante, sin negar la importancia de la aportación de estos autores, es obligado señalar que la frecuencia de la coocurrencia como factor para la detección de colocaciones ha sido muy criticada (Alonso Ramos 1994-1995:14; Corpas Pastor 2001; Sánchez Rufat 2010). La principal objeción viene dada por el hecho de que, si bien es cierto que los componentes de una colocación aparecen conjuntamente en el discurso, esta condición no resulta suficiente para determinar la existencia de estas combinaciones.

\subsection{Enfoque semántico}

De manera casi paralela a la orientación estadística surgen trabajos en los que el estudio de estos fenómenos se realiza desde posiciones que dan más peso al componente semántico. Es el caso de Coseriu (1981:152-153), quien, aun cuando no emplea en ningún momento el término colocación en relación con el concepto de solidaridad léxica, se vale del subtipo multilateral para referirse a lo que denota aquel. Esto es, la determinación que una unidad ejerce sobre otra de tal suerte que el sentido del lexema determinante está incluido en el determinado, pero no a la inversa. Así, ladrar implica a perro, igual que relinchar contiene a caballo.

Otra interpretación llega desde la Teoría Sentido-Texto (Mel'čuk 1989, 1992, 1995, 2006), que pone en práctica una interpretación de las estructuras colocacionales a través del instrumento de las funciones léxicas (FFLL), concepto creado por Mel'čuk y Žolkovskij y definido como "una herramienta especialmente diseñada para codificar elecciones léxicas

${ }^{4}$ A pesar de este reconocimiento a Firth, este autor tomó el término collocation de Harris (1957) (Koike 2001:16, Serra Sepúlveda 2012:94). 
controladas léxicamente" (Alonso Ramos 2007:452). En palabras de Bosque (2004:CLVICLVII), son:

conexiones semánticas entre palabras que permiten establecer abanicos de relaciones sumamente variadas. Las funciones léxicas definen las conexiones semánticas que existen entre el lema y cada una de las piezas que aparecen en la serie de unidades que se conectan con él.

El fruto más representativo de los postulados propuestos por estos autores es el Dictionnaire explicatif et combinatoire du français contemporain. Recherches lexicosémantiques (DEC), publicado entre 1984 y 1999. Este diccionario constituye una muestra en la que se aplica la teoría de las funciones léxicas por medio de fórmulas de difícil comprensión para un lector no formado en las teorías de esta escuela.

Con el fin de aclarar el instrumento básico del que se sirve esta teoría, recurrimos a la explicación de la función léxica (FL) Magn, que posee el valor de 'muy', 'mucho', 'intensamente'. Esta FL relaciona la base o argumento con uno o más colocativos o valores, como vemos en la Figura 1, donde se muestran las colocaciones correspondientes al español, al inglés y al francés en las que interviene la FL Magn y relaciona la base soltero con sus respectivos colocativos en las tres lenguas, empedernido, endurci y confirmed

\begin{tabular}{|c|c|c|}
\hline \multirow{2}{*}{ FUNCIÓN LÉXICA } & $\begin{array}{c}\text { BASE } \\
\text { (lexía) }\end{array}$ & $\begin{array}{c}\text { COLOCATIVO } \\
\text { (lexía o conjunto de lexías) }\end{array}$ \\
\cline { 2 - 3 } & soltero & empedernido \\
\cline { 2 - 3 } & célibataire & endurci \\
\cline { 2 - 3 } & bachelor & confirmed \\
\hline
\end{tabular}

Figura 1. Ejemplo de función léxica. Fuente: Barrios Rodríguez (2006:352).

De la mano de Alonso Ramos (1989, 1993, 1994-1995, 1997, 1999-2000, 2002, 2004a, 2004b, 2007) llega la aportación más significativa a esta corriente en el ámbito hispánico, tanto en la teoría como en la práctica. En concreto, la mayor contribución de esta autora a la Teoría Sentido-Texto y al fenómeno colocacional es el Diccionario de colocaciones del español $(\mathrm{DiCE})^{5}$. Se trata de una obra en construcción donde Alonso Ramos pone en práctica los fundamentos teóricos sobre restricciones léxicas y semánticas, ya desarrollados en sus trabajos previos. Otros estudios que pueden adscribirse a este enfoque son los de Sanromán Vilas (2000, 2009a, 2009b, 2009c, 2011).

Wotjak, en un trabajo centrado en las construcciones verbo-nominales funcionales, define la colocación como "combinación poliléxica de cierto grado de fijación" e insiste en considerar estas estructuras como "entidades socializadas y usualizadas del inventario". Si bien

\footnotetext{
${ }^{5}$ http://www.dicesp.com/paginas/index/2
} 
no las reconoce como unidades fraseológicas de facto, admite que se hallan en un proceso de lexicalización (Wotjak, 1998:258, 260).

El enfoque lexicográfico queda claramente representado por M. Benson, E. Benson y R. Ilson, compiladores de The BBI combinatory dictionary of English: A guide to word combinations (1986). En el seno de esta propuesta se distingue la colocación gramatical de la colocación léxica ${ }^{6}$, en el sentido de que la colocación gramatical se define como como "a dominant word (noun, adjective, verb) and a preposition or grammatical structure such as infinitive or clause" 7 . En palabras de Travalia (2006a:319) ${ }^{8}$, se trata de "uniones entre un elemento léxico y otro gramatical". Esta autora (2006b:285-291) lleva a cabo una revisión de la taxonomía propuesta por Benson, Benson e Ilson (1986:IX) para las colocaciones gramaticales, que, a nuestro juicio, mejora la propuesta de los británicos. La innovación de su teoría reside en su decisión de rechazar la inclusión de estructuras del tipo carecer de entre las colocaciones gramaticales. Para justificarse, analiza los rasgos tradicionalmente asociados a las colocaciones y comprueba que la posibilidad de coocurrencia no es aplicable en muchos de estos casos, dado que la presencia del elemento preposicional es obligatoria; son "estructuras sintácticas determinadas de cada lengua". Existen, sin embargo, otras combinaciones donde cabe la opción de usar el verbo con o sin preposición, incluso de intercambiar una preposición por otra; solo en estos contextos se admite la existencia de una colocación gramatical: hablar de / sobre / acerca de; abalanzarse a / contra / sobre ${ }^{9}$, por ejemplo.

También alejado de la tendencia puramente estadística hallamos el enfoque de Haensch y otros (1982:251), quienes enfatizan el hecho de que las palabras de una lengua seleccionan unidades limitadas para combinarse. Haensch (1985:238) expone como rasgo fundamental de las colocaciones su uso habitual y la ausencia de lexicalización que observa, por ejemplo, en otras estructuras, como las combinaciones fijas lexicalizadas, las expresiones fraseológicas verbales o los modismos.

La opción planteada por Gross $(1996,2014)$ se basa en la noción de clase de objetos o clase léxica, entendida esta como conjunto de unidades que muestran un comportamiento semántico-sintáctico análogo. Este autor defiende que es imprescindible conocer el esquema de argumentos de cada unidad lingüística, así como de todas aquellas que sean susceptibles de ocurrir en contextos similares con el fin de mostrar el armazón de relaciones sintáctico-semánticas existentes entre ellas. Asimismo, defiende la existencia de predicados nominales

${ }^{6}$ Koike (2001:14) explica la diferencia entre ambos tipos de colocaciones en función de su naturaleza sintáctica en el caso de las colocaciones gramaticales, y de su naturaleza semántica en lo que concierne a las colocaciones léxicas.

${ }^{7}$ La definición de estos autores ha sido comúnmente aceptada por los especialistas, si bien se hacen puntualizaciones, como la de Koike (2001:63) o revisiones, como la de Travalia (2006b:285-291).

${ }^{8}$ Dentro del subgrupo de colocaciones gramaticales, a su vez, podemos señalar una serie de subdivisiones que se corresponden con los siguientes esquemas trazados por Travalia (2006b:285-291):

Verbo + preposición: hablar de.

Sustantivo + preposición + (sustantivo / oración subordinada): miedo de.

$($ Artículo $)+$ sustantivo + preposición + (infinitivo): el derecho a .

Preposición + (artículo) + sustantivo + /preposición): contra (la) voluntad (de).

(Verbo) + adjetivo + preposición: (estar) convencido de .

(Verbo + sustantivo) + preposición + sustantivo: (anunciar algo) por los altavoces.

${ }^{9}$ Clasificación y ejemplos tomados de Travalia (2006b:285). 
que requieren el acompañamiento de verbos soporte, los cuales se han vaciado semánticamente y funcionan como meros actualizadores de aquellos.

Esta idea permite establecer una relación entre la obra de Gross y la propuesta de Bosque, quien prefiere desvincularse del término colocación, pues, a su juicio, la complejidad del concepto es mayúscula. Bosque (2004:§8, §10, §11), en el prólogo de REDES. Diccionario combinatorio del español contemporáneo, plantea que esa obra no pretende ser un diccionario de colocaciones, sino de restricciones combinatorias, que también dé cabida a otras unidades. Justifica la necesidad de recurrir a las clases léxicas basándose en que "la categorización múltiple representa la manifestación de los rasgos de una pieza léxica en los contextos que la activan", y añade que REDES da herramientas para reconstruir esos contextos. Otro aspecto de gran relevancia en su teoría es la propuesta de una selección de la base de la colocación por parte del colocativo frente a los demás enfoques, que coinciden en que es aquella la que ejerce un papel predominante sobre este.

Como representante de la corriente generativista, sobresale la obra de Mendívil Giró (1999), autor que para referirse a las colocaciones usa la voz preferencias usuales, las cuales no identifica ni con las locuciones ni con las solidaridades léxicas coserianas, pues considera que en las preferencias usuales no se da coocurrencia obligatoria de sus componentes ni hay presuposición de un miembro por la presencia de otro.

En la misma línea de Mendívil, por lo que respecta a la ubicación de las colocaciones en el punto medio del grado de fijación de las combinaciones léxicas, encontramos, desde un enfoque totalmente diferente, el estudio de Aguilar-Amat (1993:80-81), quien asume que las colocaciones se encuentran entre las combinaciones libres y las estructuras con mayor fijación, y concede un papel importante a la tradición como factor clave para que se consoliden en la lengua.

La perspectiva fraseológica tiene su mayor representación en la obra de Corpas Pastor (1996, 2001), quien, al igual que Mendívil Giró y Aguilar-Amat, establece varios grados de fijación en las combinaciones léxicas, ubicando las colocaciones en el punto medio. Sitúa estas construcciones junto a las locuciones y a los enunciados fraseológicos, dado que las considera unidades fraseológicas "de pleno derecho" al poder reconocer en ellas los siguientes rasgos distintivos: "polilexicalidad, alta frecuencia de aparición y de coaparición, institucionalización, estabilidad (fijación y especialización semántica), idiomaticidad y variación potenciales" (Corpas Pastor 2001:91).

La inclusión de las colocaciones en el ámbito fraseológico se reconoce también en la obra de Castillo Carballo (2001:268-269), quien les atribuye las cualidades de la composicionalidad y de la idiomaticidad, fundamentales para establecer la divergencia con las locuciones y con las combinaciones libres, respectivamente. Destaca, asimismo, el interés de la autora por la aplicación lexicográfica de las colocaciones, señaladamente en el ámbito de la enseñanza de segundas lenguas.

Una de las aportaciones más relevantes del estudio de las colocaciones, centrada en el español, es la obra de Koike (1993, 1995, 1996a, 1996b, 1997, 1998, 2001, 2002, 2005, 2006, 2008). En su monografía de 2001 lleva a cabo una exhaustiva caracterización de las colocaciones, exponiendo a lo largo de ella las ideas más relevantes de las teorías tradicionales para 
corroborarlas, rechazarlas o matizarlas. Su estudio se aleja de la perspectiva estadística y se ubica en la órbita de los trabajos de corte semántico. Constituye, en la actualidad, el trabajo más completo dedicado en exclusiva a las colocaciones.

\section{ESTUDIOS DIACRÓNICOS}

Ya sea por el auge relativamente reciente del estudio de las colocaciones, que hace necesaria una precisión de los atributos básicos de estas construcciones, ya sea por la dificultad añadida de aplicar estas nociones en materiales con menor accesibilidad, los estudios sobre ellas desde la perspectiva histórica ${ }^{10}$ no han sido frecuentes.

En los trabajos sobre el español podemos citar la aportación de García Pérez (2007c), quien realiza un recorrido histórico de las construcciones con verbo de apoyo en español en las que interviene hacer desde el siglo XII hasta el siglo XX. El autor mantiene, siguiendo a Piera y Varela (1999:4415-4418), que las combinaciones de este tipo son colocaciones sometidas a un alto grado de lexicalización. Su clasificación, si bien coincide con otras de las ya citadas en aspectos como la arbitrariedad de la selección del colocativo por parte de la base, retoma el concepto de clase léxica ${ }^{11}$ e incide en el estudio del léxico con una perspectiva relacional.

Desde una corriente teórica distinta a la adoptada por García Pérez, pero coincidente en la perspectiva diacrónica, sobresalen los trabajos de Alba-Salas ${ }^{12}$ (2002, 2006, 2007, 2009, 2011, 2012a, 2012b, 2013a, 2013b). La obra de este autor, para quien las colocaciones se hallan a medio camino entre los sintagmas libres y las expresiones idiomáticas, constituye una gran contribución al estudio histórico de las colocaciones del español. Sus exhaustivas investigaciones sobre las combinaciones verbo + sustantivo en función de objeto directo basadas en los materiales del Corpus del Español de Davies se fundamentan en la Teoría Sentido-Texto. No obstante, no pone énfasis en el estudio abstracto de las funciones léxicas que prima en los trabajos de Alonso Ramos. Por el contrario, la orientación práctica de sus trabajos se percibe en dos características fundamentales. En primer lugar, destaca la extraordinaria importancia concedida al estudio del corpus, que aparece tras unas sólidas — y sintéticas — indicaciones teóricas y se convierte en el protagonista. Por otra parte, además de los datos puramente lingüísticos, el autor ofrece una investigación de posibles causas socio-históricas que propician los cambios señalados. Se suma, de este modo, a la nómina de especialistas (Gerritsen y Stein 1992; Company 2006, 2009; Espinosa Elorza 2010:14) que subrayan la importancia de relacionar los factores internos y externos en el estudio histórico de la lengua.

Metodológicamente, los trabajos de Alba-Salas —en su mayoría centrados en parejas de verbos que compiten durante el recorrido histórico de una colocación-constituyen una síntesis

\footnotetext{
${ }^{10}$ Aun partiendo de la menor cantidad de trabajos diacrónicos, contamos, fuera del ámbito hispánico con las aportaciones de Chaurand (1983), La Fauci (1972) y Marchello-Nizia (1996).

${ }^{11}$ García Pérez maneja la noción de clase léxica en otros trabajos (García Pérez, 2006:317; 2007a:63-74, 2007b:75-86).

12 En Alba-Salas (2006:45-57) se realiza una aproximación a las colocaciones desde un enfoque teórico. Se les atribuye una serie de rasgos que permiten distinguirlas de otras combinaciones en función del grado de fijación de sus componentes.
} 
entre el modelo propugnado por la Teoría Sentido-Texto que aplica en sus trabajos Alonso Ramos y el sistema de clases léxicas defendido por García Pérez y por Bosque. Este último enfoque entiende el estudio del léxico como un entramado de relaciones que son capaces de dar cuenta de la naturaleza lingüística de un idioma mucho mejor que la investigación aislada de las voces que lo integran. Así, cuando Alba-Salas se propone evaluar la trayectoria histórica de la colocación dar miedo, también forman parte de su indagación otros sustantivos semánticamente relacionados con la base de la colocación mencionada. En consecuencia, en muchas ocasiones, al investigar sobre la clase léxica, y no solo sobre la voz que constituye la colocación en cuestión, la información de carácter lingüístico - y extralingüístico — se ramifica y multiplica, y permite trazar con mayor precisión el origen, la evolución y la muerte de las combinaciones.

\section{ACERCAMIENTO A UNA DEFINICIÓN DE COLOCACIÓN Y A UNA SISTEMATIZACIÓN DE SUS RASGOS CARACTERIZADORES}

A partir de la revisión de las diferentes teorías que ponen el foco de atención en las colocaciones, llegamos a la conclusión de que se trata de combinaciones léxicas en las que intervienen dos elementos. La diferencia fundamental entre ambos es que uno de ellos, la base, es elegido de manera intencional por el hablante. Por contra, la selección del segundo elemento, el colocativo, no es voluntaria, sino fruto de una preferencia por parte de la base en función de las relaciones semánticas y, especialmente, de los factores extralingüísticos que confluyen y son determinantes en el triunfo de una unidad u otra. De esta manera, en la estructura dar un beso, la base sería el sustantivo beso, puesto que constituye el componente con mayor carga semántica. El verbo, sin embargo, podría ser sustituido por otros de sentido similar sin que el valor de la construcción se viera profundamente alterado. La preferencia hacia él que muestra la base es arbitraria.

Precisamente, es la arbitrariedad en la selección del colocativo una de las características de las colocaciones que las contraponen a otras estructuras más fijas, como son las locuciones. No se trata de una imposición sintáctica ni semántica, sino una elección que obedece a criterios extralingüísticos (Luque y Manjón 1997:17; Alba-Salas 2006:47), entre los que sobresale la consolidación forjada por el uso de la comunidad de hablantes; se entiende que las colocaciones se transmiten como construcciones "previamente hechas" (Zuluaga 2001:71). Esta falta de motivación queda probada con el hecho de que existan bases con significados cercanos que seleccionan colocativos distintos. Alba-Salas (2006:47) aduce los ejemplos de los sustantivos aviso y advertencia, que poseen un sentido similar y se combinan, respectivamente, con los verbos dar y hacer.

Otro argumento que refuerza esta idea es la elección de distintos colocativos según el idioma, pues si fuera una razón lingüística la que obliga que en español la colocación sea prestar atención, debería tener su correspondencia con el inglés *lend attention, en lugar de pay attention.

Existe, asimismo, variación diatópica en las colocaciones. Dependiendo del lugar, aunque se mantenga una base común, el colocativo cambia. Es el caso del par de colocaciones dimisión indeclinable y dimisión irrevocable, características del español de Chile y de España, respectivamente (Koike, 2001:201). 
Por último, cabe aludir a la variación diacrónica, pues observamos que una base puede seleccionar distintos colocativos a lo largo de la historia de una lengua. Así, los sustantivos que denotan el golpe dado con un arma, del tipo de cuchillada, coaparecen con los verbos dar y tirar (Martos García, en prensa). Véanse algunos ejemplos extraídos del Corpus diacrónico del Reino de Granada (Calderón Campos y García Godoy, 2014):

1. el dicho don Cristóbal se vino contra ellos con su espada tirándoles munchas cuchilladas con intento de matarles (1600 CORDEREGRA, ARCHGR: 9370).

2. puso mano a la espada que llevaba y, desnuda, tiró muchos golpes y cuchilladas a los criados de los caballeros y personas que habían acompañado al dicho doctor Rodrigo de Mújica y, en particular, le dio una cuchillada en la cabeza a un esclavo del dicho Andrés Bravo Suárez (1623 CORDEREGRA, ARCHGR: Málaga, 9633/7).

3. y con sus espadas desnudas, el uno por delante y el otro por detrás, le tiraron muchas cuchilladas y estocadas para matarle, como lo hicieran si no se defendiera porque de uno cuchillada [sic] le rompieron el ferreruelo por detrás y le dieron en el sombrero otras dos cuchilladas y le hirieron en un dedo, de que le salió mucha sangre (1625 CORDEREGRA, ARCHGR: 9658/7).

4. el dicho Juan de Castañeda sacó la espada y fue hacia el dicho Marcos de Zaragoza según echó de ver después este testigo, porque llegó y por detrás le dio una guchillada [sic] al dicho Marcos de Zaragoza en la cabeza (1627 CORDEREGRA, ARCHGR: 10050/15).

Aunque el uso del verbo dar predomina sobre el de tirar desde el siglo XVI al siglo Xx, se observa, tanto en los ejemplos mostrados como en los que ofrece el CORDE, que en las dos primeras centurias se produjo una aproximación en el empleo ambos verbos, de modo que llegaron a utilizarse en contextos similares. La elección de un elemento u otro, no obstante, responde al matiz semántico que persigue transmitir la construcción: considerando ambos verbos como un par, tirar es el elemento no marcado, del cual se sirve el hablante para expresar la acción de golpear con un arma, sin especificar que el ataque resulte efectivo y traiga consigo la herida del contrario. Por el contrario, dar, elemento marcado, además de expresar la acción de ejecutar el golpe, implica alcanzar el blanco.

Otra característica propia de las colocaciones es la restricción combinatoria. El uso que la comunidad lingüística hace de estas estructuras se convierte en un factor determinante para su fijación, de modo que acaban siendo las escogidas entre otras opciones igualmente válidas desde el punto de vista gramatical o léxico, razón por la que Koike (2001:27) habla de "predilección léxica", mientras que Írsula prefiere "atracción preferente" (1994:277) para referirse a la relación que existe entre una base y un colocativo concretos. La selección que tiene lugar en este tipo de combinaciones, consolidada por la recurrencia de su empleo, está fijada en la norma, no en el sistema (Írsula 1994:279; Corpas Pastor 1996:53; Koike 2001:27). Se trata de un criterio reconocido ampliamente por los especialistas de diferentes enfoques teóricos (Írsula 1994:279; Corpas Pastor 1996:53; Mendívil Giró 1991:717; Koike 2001:27). Así, pues, aun cuando no todos coinciden en insertar las colocaciones dentro de la fraseología, es innegable que existe una fuerte tendencia de la base a escoger un colocativo frente a otros con los que puede ofrecer combinaciones de características semánticas casi idénticas ${ }^{13}$. Es este rasgo uno

${ }_{13}$ Valga el ejemplo aducido por Koike (2001:27) de la colocación superar una dificultad, en la que la base dificultad puede combinarse con otros colocativos como salvar o vencer. 
de los que permiten establecer diferencias entre las colocaciones y las locuciones, en las que no cabe sustituir alguno de sus componentes por términos sinónimos. Comprobamos que es inviable este proceso en el reemplazo de *arrojar de menos por echar de menos.

En tercer lugar, destaca la composicionalidad de las colocaciones. Pese a este papel semántico preponderante que reconocemos en la base, no se produce una fusión del significado de los dos elementos resultante en un valor unitario de la construcción. Por el contrario, asistimos a la combinación entre dos unidades que añaden su valor a la estructura que conforman (Cruse 1986:40; Cumming 1986:19; Calderón Campos 1994:81), de modo que son combinaciones con una posible variedad combinatoria y morfológica (Mendívil Giró 1991:717) ${ }^{14}$. Este rasgo aleja las colocaciones de las locuciones, pues en estas últimas no expresan un sentido basado en la suma de los elementos que las integran. Así, el significado de beber los vientos (por alguien) no resulta de la suma de los valores del verbo y del sustantivo.

\section{CONCLUSIONES}

Tras la revisión teórica que hemos presentado, comprobamos cómo las estructuras colocacionales han despertado el interés de especialistas adscritos a diversos enfoques teóricos desde las últimas décadas del siglo xx en el ámbito hispánico. Diferenciamos, por un lado, aquellos autores que interpretan las colocaciones en clave estadística por la frecuencia de coaparición de los elementos que las integran y, por otro, los que comprenden estas estructuras poniendo el peso en los matices semánticos.

A nuestro juicio, dicho criterio no constituye una particularidad que posibilita el reconocimiento de las colocaciones. Siguiendo a Wotjak (1998:260-262), estimamos que, aunque esta coocurrencia de los elementos tiene lugar en muchas ocasiones, no podemos fundamentar la existencia de una colocación en la coocurrencia frecuente de sus elementos. Por ende, defendemos que lo que determina la existencia de una colocación son las relaciones de tipo semántico y sintáctico que existen entre la base y el colocativo.

Por lo que respecta a la atención dedicada a las colocaciones en el terreno de la lengua española, advertimos un desajuste profundo entre las investigaciones de corte sincrónico y los estudios diacrónicos, ya que frente al primer grupo, que cuenta con trabajos de variada extensión, incluso con obras lexicográficas, el segundo no cuenta con una representación nutrida. Esta situación revela la necesidad de ahondar en el estudio histórico de las colocaciones, pues sería determinante para enriquecer el conocimiento de estas estructuras en la actualidad y proporcionaría herramientas útiles para llevar a cabo estudios contrastivos de distintas lenguas.

\footnotetext{
${ }^{14}$ Es necesario apuntar que la composicionalidad semántica ha sido puesta en duda (Írsula 1994:277), pues existen casos de colocaciones determinadas por una lexicalización más fuerte (Wotjak 1998:258), que provoca que la fijación sea mucho mayor e imposibilita la transparencia semántica que cifran como atributo de las colocaciones, entre otros, Hausmann (1989:1010) o Bahns (1993:57). Para Alonso Ramos (1994-1995:24), lo más adecuado es establecer una gradación en la composicionalidad que permita diferenciar construcciones. De este modo, encontramos combinaciones libres, completamente composicionales; colocaciones, parcialmente composicionales; y frasemas, no composicionales.
} 
Después del estudio del horizonte epistemológico de las colocaciones, definimos estas estructuras como combinaciones léxicas en las que intervienen dos elementos: la base, que se elige de manera intencional, y el colocativo, cuya selección no es voluntaria, sino que obedece a restricciones semánticas y también extralingüísticas. Entre los rasgos más representativos de las colocaciones sobresalen: a) la arbitrariedad en la selección del colocativo, que queda probada con la existencia de variantes diatópicas y diacrónicas, b) la restricción semántica entre los dos componentes de la colocación, y c) la composicionalidad perceptible entre la base y el colocativo, ausente en construcciones más fijas, como las locuciones.

\section{REFERENCIAS BIBLIOGRÁFICAS}

Aguilar-Amat, Anna (1993): "En torno a la combinatoria del léxico: los conceptos de colocación e idiomatismo", en Martín Vide, Carlos (coord.), Lenguajes naturales y lenguajes formales: actas del IX congreso de lenguajes naturales y lenguajes formales: (Reus, 20-22 de diciembre de 1993), Barcelona, PPU, pp. 267-272.

Alba-Salas, Josep (2002): Light Verb Constructions in Romance: A Syntactic Analysis, Nueva York, Ithaca.

- (2006): "Las colocaciones con nombre predicativo: consideraciones prácticas y metodológicas para su tratamiento lexicográfico", en Alonso Ramos, Margarita (coord.), Diccionarios y fraseología, A Coruña: Universidade da Coruña, pp. 45-58.

- (2007): "On the Life and Death of a Collocation: A Corpus-Based Diachronic Study of dar miedo/ hacer miedo-type Structures in Spanish", Diachronica, 24, pp. 207-252.

- (2009): "Las estructuras tipo meter miedo en diacronía: Más detalles sobre la evolución histórica de las colocaciones causativas", en Enrique Arias, Andrés (ed.), Diacronía de las lenguas iberorrománicas: Nuevas aportaciones desde la lingüística de corpus, Madrid / Frankfurt, Iberoamericana / Vervuert, pp. 343-364.

- (2011): "Realineamiento paradigmático en las estructuras reiterativas con sustantivos de cualidad y estado: cobrar, recobrar, y recuperar en diacronía", Lenguaje, 29, pp. 333-362.

- (2012a): "Colocaciones incoativas con "tomar" y "prender" en diacronía", Revista de Historia de la Lengua Española, 7, pp. 3-38.

- (2012b): "The Origins and Evolution of Inchoative Collocations with dar in Spanish", Romance Philology, 66, pp. 363-393.

- (2013a): "De 'recuperar' a 'adquirir': Sobre la evolución histórica de las colocaciones tipo cobrar afecto y cobrar importancia", Romanische Forschungen, 125, pp. 151-193.

- (2013b): "Cobrar miedo: sobre el uso histórico de cobrar en colocaciones con cualidades y estados negativos", Scriptum Digital, 2, pp. 77-106.

Alonso RAMOS, MARgaRita (1989): "Aproximación a un nuevo modelo lexicográfico: el diccionario explicativo y combinatorio del francés contemporáneo", Anuario Galego de filoloxía, 16, pp. 421-450.

- (1993): Las funciones léxicas en el modelo lexicográfico de I. Mel'čuk. Madrid, UNED.

- (1994-1995): "Hacia una definición del concepto de colocación: de J. R. Firth a I. A. Mel'čuk", Revista de lexicografía, I, pp. 9-28.

- (1997): "Coocurrencia léxica y descripción lexicográfica del verbo DAR: hacia un tratamiento de los verbos soportes", Zeitschrift fur romanische philologie, 113(3), pp. 380-417.

- (1999-2000): "Verbos de apoyo, funciones léxicas y traducción automática", Revista de Lexicografía, 6, pp. 155-177.

- (2002): "Colocaciones y contorno de la definición lexicográfica", LEA: Lingüística Española Actual, XXIV(1), pp. 63-96. 
Alonso Ramos, Margarita (2004a): "Elaboración del Diccionario de colocaciones del español y sus aplicaciones", en Battaner, Paz y De Cesaris, Janet (eds.), De Lexicografia. Actes del I Simposium internacional de Lexicografía, Barcelona, IULA y Edicions Petició.

- (2004b): Las construcciones con verbo de apoyo, Madrid, Visor Libros.

- (2007): "Actantes y colocaciones". Nueva Revista de Filología Hispánica, LV(2), pp. 435-458.

BAHns, Jens ( I993): "Lexical collocations: a contrastive view", ELT Journal, 47/1, pp. 56-63.

Barrios Rodríguez, María Auxiliadora (2006): "Macroestructura y microestructura de un diccionario explicativo y combinatorio: diferencias con respecto a un diccionario común", Revista española de lingüística, 36, 1, pp. 349-378.

Benson, Morton, Benson, Evelyn e Ilson, Robert (1986): The BBI Combinatory Dictionary of English: A Guide to Word Combinations, Amsterdam, John Benjamins.

Bosque MuÑoz, Ignacio (dir.) (2004): REDES. Diccionario combinatorio del español contemporáneo, Madrid, SM.

Calderón Campos, Miguel (1994): Sobre la elaboración de diccionarios monolingües de español, Granada: Ediciones Universidad de Granada.

Calderón Campos, Miguel, y García-Godoy, María Teresa (dirs.) (2014): Corpus diacrónico del español del Reino de Granada. 1492-1833. http://www.corderegra.es.

Castillo Carballo, María Auxiliadora (2001): "Norma y producción lingüística: las colocaciones léxicas en la enseñanza de segundas lenguas", en Martín Zorraquino, María Antonia y Díez Pelegrín, Cristina (eds.), ¿Qué español enseñar?: norma y variación lingüisticas en la enseñanza del español a extranjeros: actas del XI Congreso Internacional ASELE, Zaragoza 13-16 de septiembre de 2000, Zaragoza, Universidad de Zaragoza, pp. 267-272.

Chaurand, Jacques (1983): 'Les verbes supports en ancien français: 'donner' dans les oeuvres de Chrétien de Troyes", Lingvisticae Investigationes, 7(2), pp. 11-47.

Company Company, Concepción (dir.). (2006): Sintaxis histórica de la lengua española. Primera parte: La frase verbal, 2 vols., México, Fondo de Cultura Económica y Universidad Nacional Autónoma de México.

- (dir.). (2009): Sintaxis histórica de la lengua española. Segunda parte: La frase nominal, 2 vols., México: Fondo de Cultura Económica y Universidad Nacional Autónoma de México.

Corpas Pastor, Gloria (1996): Manual de fraseología española, Madrid, Gredos.

- (2001): "Apuntes para el estudio de la colocación”, LEA: Lingüística española actual, 23 (1), pp. $41-56$.

- (2003): Diez años de investigación en fraseología: análisis sintáctico-semánticos, contrastivos y traductológicos, Madrid, Vervuert.

Coseriu, Eugenio (1981): "Las solidaridades léxicas”, Principios de semántica estructural, Madrid, Gredos.

Cruse, David Alan (1986): Lexical semantics, Cambridge, Cambridge University Press.

Cumming, Susanna (1986): The lexicon in text generation, California, Information Sciences Institute, University of Southern California.

Espinosa Elorza, Rosa María (2010): Procesos de formación y cambio en las llamadas "palabras gramaticales”, San Millán de la Cogolla, Cilengua.

Ferrando Aramo, Verónica (2012): Aspectos teóricos y metodológicos para la compilación de un diccionario combinatorio destinado a estudiantes de E/LE, Barcelona, Universitat Rovira y Virgili.

FIRTH, John (1957): Papers in linguistics 1934-1951, Londres, OUP.

García Pérez, Rafael (2006): "El proceso de formación de las clases léxicas y su importancia para un diccionario histórico: el ejemplo de la clase <odio>”, Boletín de la Real Academia Española (BRAE), tomo LXXXVI, Cuaderno CCXCIV, pp. 317-332.

- (2007a): “¿Desde cuándo se cometen delitos?”, en Pascual, José Antonio y García Pérez, Rafael, Límites y horizontes en un diccionario histórico, Salamanca, Diputación de Salamanca, pp.68-74. 
García Pérez, Rafael (2007b): “¿Desde cuándo se imponen penas?”, en Pascual, José Antonio y García Pérez, Rafael, Límites y horizontes en un diccionario histórico, Salamanca, Diputación de Salamanca, pp. 75-86.

- (2007c): ¿Qué hacíamos y qué hacemos?: el verbo hacer en la historia del español, San Millán de la Cogolla, Cilengua.

García-Page SÁnchez, Mario (2001): “¿Son las expresiones fijas expresiones fijas?”, Moenia, 7 , pp. 165-197.

Gerritsen, Marinel y Stein, Dieter (eds). (1992): Internal and External Factors in Syntactic change, Berlin, Mouton de Gruyter.

Gómez Molina, José Ramón (2004): "Las unidades léxicas en español”, Carabela, 56, pp. 27-50.

Gross, Gaston (1996): Les expressions figées en français: noms composés et autres locutions, Gap-Paris, Ophrys.

- (2014): Manual de análisis lingüístico. Aproximación sintáctico-semántica al léxico, Barcelona, Editorial UOC.

Haensch, Gunther (1985): "La selección del material léxico para diccionarios descriptivos", en Fernández-Sevilla, Julio et al. (coords.), Philologica Hispaniensia in Honorem Manuel Alvar II, Madrid, Gredos, pp. 227-254.

HAENSCH, GunTher et al. (1982): La lexicografía, Madrid, Gredos.

Halliday, Michael (1961): "Categories of the Theory of Grammar", Word, 17, pp. 241-92.

- (1966): "Lexis as a Linguistic Level", en Bazell, Charles et al (eds.), In Memory of J.R. Firth, London: Longman, pp.148-63.

Harris, Zellig (1957): "Co-Occurrence and Transformation in Linguistic Structure”, Language, 33, 3, pp. 283-340.

Hausmann, Franz Josef (1989): "Le dictionnaire de collocations", en Hausmann, Franz Josef et al. (eds.), Wörterbücher /Dictionaries / Dictionnaires (Ein internationales Handbuch zur Lexikographie/ An International Encyclopedia of Lexicography/ Encyclopédie internationale de lexicographie, vol. 1, Berlín, Nueva York: Walter de Gruyter, pp. 1010-1019.

ÍRSUlA PeÑA, Jesús (1994): "Entre el verbo y el sustantivo, ¿quién rige a quién? El verbo en las colocaciones sustantivo-verbales", en Endruschat, Annette et al. (coords.), Verbo e estruturas frásicas. Actas do IV Colóquio Internacional de Linguística Hispânica de Leipzig, Oporto: Universidad de Oporto, pp. 277-286.

Koike, Kazumi (1993): “Caracterización y estructuras del verbo compuesto”, Hispánica, (37), pp. 14-29.

- (1995): "Verbos soporte con valor aspectual”, Hispánica, 39, pp. 88-101.

- (1996a): "Verbos funcionales en español", en Ueda, Hiroto (coord.), Homenaje al Prof. Makoto Hara, Tokio: Universidad de Estudios Extranjeros de Tokio, pp. 161-179.

- (1996b): "Verbos colocacionales en español", Hispánica, 40, pp. 14-31.

- (1997): Alcance y características de las colocaciones sustantivo-verbales en español, Memoria de Investigación, Madrid, UNED.

- (1998): "Algunas observaciones sobre colocaciones sustantivo-verbales", en Wotjak, Gerd (ed.). Estudios de fraseología y fraseografía del español actual, Madrid / Frankfurt, Iberoamericana / Vervuert, pp. 245-255.

- (2001): Colocaciones léxicas en el español actual: estudio formal y léxico- semántico, Alcalá de Henares, Universidad de Alcalá de Henares.

- (2002): "Comportamientos semánticos en las colocaciones léxicas", LEA: Lingüística española actual, 1(24), pp. 5-24.

- (2005): "Colocaciones complejas en el español actual”, en Almela, Ramón, Wotjak, Gerd y Ramón Trives, Estanislao (coords.), Fraseología contrastiva: con ejemplos tomados del alemán, español, francés e italiano, Murcia: Servicio de Publicaciones de la Universidad de Murcia, pp. 169-184. 
Koike, Kazumi (2006): "Colocaciones metafóricas", en De Miguel Aparicio, Elena, Serradilla Castaño, Ana y Palacios Alcaine, Azucena (coords.), Estructuras léxicas y estructura del léxico, Alemania: Peter Lang, pp. 47-60.

- (2008): "Colocaciones atípicas", LEA: Lingüística española actual, 30 (1), pp. 87-107.

La Fauci, Nunzio (1972): "Aspects du mouvement de wh-, verbes supports, double analyse, complétives au subjonctif en italien: pour une description compacte", Lingvisticae Investigationes, 4(2), pp. 293-341.

Luque Durán, Juan De Dios, y Manjón Pozas, Francisco José (1998): “Colocaciones léxicas: cuestión lingüística o estilística”, en Luque Durán, Juan de Dios, y Manjón Pozas, Francisco José (eds.), Teoría y práctica de la lexicología: $V$ Jornadas internacionales sobre estudio y enseñanza del léxico, Granada 1997, Granada, Método Ediciones, pp. 15-24.

Marchello-Nizia, Christiane (1996): "Les verbes supports en diachronie. Le cas du français", Langages, 121, pp. 91-98.

Martos García, Paula (en prensa): “Hasta cuándo se tiran estocadas en español? Análisis de la colocación «tirar + sustantivos que denotan el golpe dado con un arma”, en Sánchez Méndez, Juan (coord..), Actas del Segundo Congreso Internacional Tradición e Innovación: nuevas perspectivas para la edición, la investigación y el estudio de documentos antiguos, celebrado en Neuchâtel entre el 7 y el 9 de septiembre de 2011.

Mel'čuK, Igor (1989): "Semantic primitives from the viewpoint of the Meaning-Text Linguistic Theory", Quaderni di Semantica, 10, 1, pp. 65-101.

- (1992): "Paraphrase et lexique: la théory Sens-Texte et le Dictionnaire explicatif et combinatoire", en Mel'čuk, Igor et al., Dictionnaire explicatif et combinatoire du français contemporain. Recherches lexico-sémantiques III, Montréal: Les Presses de L'Université de Montréal, pp. 9-58.

- (1995): "Phrasemes in Language and phraseology in Linguistics", en Everaert, Martin et al. (eds.), Idioms: Structural and Psychological Perspectives, New Jersey: Lawrence Erlbaum Associates, pp. 167-233.

- (2006): Colocaciones en el diccionario. En Alonso Ramos, M. Diccionarios y fraseología (pp. 1144). A Coruña: Universidade da Coruña.

Mendívil Giró, José Luis (1991): “Consideraciones sobre el carácter no discreto de las expresiones idiomáticas", En Martín Vide, Carlos (coord.), Lenguajes naturales y lenguajes formales: actas del VI congreso de lenguajes naturales y lenguajes formales, Barcelona, PPU, pp. 711-736.

- (I999): Las palabras disgregadas. Sintaxis de las expresiones idiomáticas y los predicados complejos, Zaragoza, Prensas Universitarias de Zaragoza.

Moreno Pereiro, Sabela y Buyse, Kris (2003): “Colocaciones léxicas: pistas y trampas”, Mosaico, 10, pp. 10-17.

MuÑIz Álvarez, Eva María (2002): “Las colocaciones como combinaciones léxicas libres”, en Veiga Rodríguez, Alexandre et al. (eds.), Léxico y gramática, Lugo: Tris Tram, pp. 261-271.

Navarro, Carmen (2003): "Didáctica de las unidades fraseológicas", en Calví, Maria Vittoria y Félix San Vicente (eds.), Didáctica del léxico y nuevas tecnologías, Viareggio: M. Baroni, pp. 99-115.

Pazó Espinosa, José y Travalia, Carolina (2008): "Las relaciones paradigmáticas en la enseñanza de las colocaciones en E/LE", en Monroy, Rafael y Sánchez, Aquilino (eds.), 25 años de lingüística aplicada en España: hitos y retos, Murcia: Universidad de Murcia, pp. 395-402.

Piera, Carlos y Varela, Soledad (1999): "Relaciones entre morfología y sintaxis", en Bosque, Ignacio y Demonte, Violeta (eds.), Gramática descriptiva de la lengua española, Madrid, Espasa Calpe, pp. 4367-4422.

SÁnchez Rufat, ANna (2010): "Apuntes sobre las combinaciones léxicas y el concepto de colocación”, Anuario de Estudios Filológicos, XXXIII, pp. 291-306.

SANROMÁn Vilas, Begoña (2000): "Presentación de una entrada lexicográfica para un diccionario explicativo y combinatorio: “esperanza”, en Pamiés Bertrán, Antonio y Luque Durán, Juan de 
Dios (coords.), Trabajos de lexicología y fraseología contrastivas, Granada, Método Ediciones, pp. 277-286.

- (2009a): "Del sentido a la expresión: los actantes en una clase de predicados nominales", en Fant Lars et al. (eds.), Actas del II Congreso de Hispanistas y Lusitanistas Nórdicos: Actas do II Congresso de Hispanistas e Lusitanistas Nórdicos (246-259). Estocolmo: Universidad de Estocolmo.

- (2009b): "Diferencias semánticas entre construcciones con verbo de apoyo y sus correlatos verbales simples", ELUA: Estudios Lingüísticos de la Universidad de Alicante 23, pp. 291-316.

- (2009c): "Towards a semantically oriented selection of the values of Oper1. The case of golpe 'blow' in Spanish", en Beck, David et al. (eds.), Proceedings of the Fourth International Conference on the Meaning-Text Theory (MTT2009), Montreal, Observatoire de Linguistique Sens-Text, pp. pp. 327-337.

- (2011): “¿Cuando hago una promesa, prometo? Límites parafrásticos con predicados de lengua”, Nueva revista de filología hispánica, 59(2), pp. 369-418.

Serra Sepúlveda, Susana (2010): "Colocaciones y solidaridades léxicas. Un intento de delimitación conceptual", Lexi-lexe, 10, pp. 1-25.

- (2012): Gramática y diccionario: contornos, solidaridades léxicas y colocaciones en lexicografía española contemporánea. Tesis doctoral, Madrid, Universidad Complutense de Madrid.

Sinclair, John (1966): "Beginning the Study of Lexis", en Bazell, Charles et al., In Memory of J.R. Firth, Londres: Longman, pp.410-31.

- (1991): Corpus, Concordance, Collocation. Oxford, Oxford University Press.

Travalia, Carolina (2006a): "Las colocaciones implícitas", Estudios de lingüística, 20, pp. 317-334.

- (2006b): "Las colocaciones gramaticales en español", Anuario de estudios filológicos, 29, pp. 279-293.

WotjaK, Gerd (1998): "Reflexiones acerca de construcciones verbo-nominales funcionales", en Wotjak, Gerd (coord.), Estudios de fraseología y fraseografía del español actual, Madrid / Frankfurt: Iberoamericana / Vervuert, pp.257-280.

Zuluaga Ospina, Alberto (2001): "Análisis y traducción de unidades fraseológicas desautomatizadas", PhiN, 16, pp. 67-83. 
